\title{
Ventricular factors linked to early and late post- cardiac surgery mortality
}

Published online: 22 August 2012

(C) Springer Healthcare 2012

medwireNews: Increased left ventricular mass index (LVMI) predicts perioperative mortality in patients undergoing cardiac surgery, while a decreased fractional area of contraction (FAC) in the left ventricle is linked to 1-year mortality, US scientists have found.

The team notes that perioperative management of patients with increased LVMI should therefore be paid special attention and write in the British Journal of Anaesthesia: "While this single study is not sufficient to conclude that LVMI should replace LV function in preoperative cardiac surgery scoring systems, it will perhaps stimulate further research in this area."

They add: "Further studies are also required to address whether 30 day mortality could be improved by performing surgery before significant increases in LVMI occur."

Led by Menachem Weiner, from the Mount Sinai School of Medicine in New York, the researchers examined intraoperative transesophageal echocardiography images of 844 cardiac surgical patients. They found that the LVMI was increased in 473 (56.4\%) patients, with 101 classified as having mildly abnormal LVMI, 106 as moderately abnormal LVMI, and 266 severely abnormal LVMI.

With 28 (3.3\%) patients having died at 30 days, the team found that there was an almost linear relationship between increasing LVMI and the risk for mortality within 30 days of surgery. Multivariate analysis that controlled for age, weight, gender, surgery type, LV function, and functional status showed that LVMI was an independent predictor for 30-day mortality, at odds ratios of 1.36 per $20 \mathrm{~g} / \mathrm{m}^{2}$ increase in LVMI, 2.53 per $60 \mathrm{~g} / \mathrm{m}^{2}$ increase, and 4.71 per $100 \mathrm{~g} / \mathrm{m}^{2}$ increase.

The researchers also report that 91 (10.8\%) patients died within 1 year of surgery. Multivariate analysis demonstrated that FAC was an independent predictor for postoperative mortality at 1 year, at odds ratios of 5.42 for a severe grading and 2.16 for a moderate grading compared with a normal FAC grading.

By Liam Davenport, medwireNews Reporter

\section{Reference}

Br J Anaesthesia 2012; Advance online publication 